\title{
Chain-Extended Polyethylene
}

\author{
P. J. Lemstra, N. A. J. M. van Aerle, \\ and C. W. M. BASTIAANSEN \\ Department of Polymer Technology, Eindhoven University of Technology, \\ P. O. Box 513, 5600 MB Eindhoven, The Netherlands
}

(Received August 28, 1986)

\begin{abstract}
The current status of high-strength/high-modulus polyethylene fibres is reviewed with the emphasis on fundamental aspects of ultra-drawing and processability. The drawing mechanism as well as the temperature dependence of the drawability of UHMW-PE (ultra-highmolecular-weight polyethylene) will be discussed.

KEY WORDS Polyethylene Fibres / Gelspinning / Ultra-Drawing Drawing Mechanisms / Strength / Modulus / WAXS / SAXS / SANS / Hexagonal Phase /
\end{abstract}

In the past ten years, great progress has been made towards the development and production of high-strength/high-modulus organic fibers. Two major routes can be discerned which are completely different with respect to the starting materials, respectively based on intrinsically rigid vs. intrinsically flexible macromolecules.

In the case of rigid chain molecules, one of the prime prerequisites for obtaining highmodulus fibrous structures: "chains perfectly ordered in an array with the chain-axis parallel to the fiber direction," 1 is met relatively easily. Examples of completely rigid chain molecules are PBT [poly $(p$-phenylenebenzobisthiazole $)]$ and its molecular analogue PBO $[\operatorname{poly}(p-$ phenylenebenzobisoxazole)]. Their persistence length $P$, as inferred from light scattering measurements in dilute solutions, is identical ${ }^{2}$ within experimental error with their contour length $L$. The specific strength and stiffness values of PBT fibres are indicated in Figure 1.

Well known fibres based on "rigid" chain molecules are the aromatic polyamides (aramids) notably PPTA [poly ( $p$-phenyleneterephthalamide)], currently marked under the trade names of Kevlar (du Pont) and Twaron (AKZO/ENKA). In contrast to PBT and
PBO, the aromatic polyamide chains are not strictly rigid, the $L / P$ ratio of PPTA is about 4 in dilute solutions. ${ }^{3}$ Spinning conditions have been found however which promote chain-extension and alignment, such as processing/spinning from nematic solutions, controlled coagulation and heat-setting under tension. The specific values for tenacity and modulus of PPTA fibres are shown in Figure 1.

Flexible/regular macromolecules such as polyethylene, polypropylene and polyesters/ amides, tend to fold during crystallization/ solidification from the melt and solutions. Consequently in conventional spinning procedures such as melt-spinning or solutionspinning, either wet or dry, folded-chain type crystals will be present in the as-spun yarns. Their prevalence will depend upon their molecular characteristics (chain-length, branchcontent, stereoregularity), type of solvent and spinning conditions. The main problem, in comparison to spinning of rigid chain polymers, is to transform these folded-chain type crystals into chain-extended structures in order to exploit the intrinsic properties of the polymer chain with respect to stiffness and strength. In the case of flexible macromolecules, polyethylene has been studied extensive- 


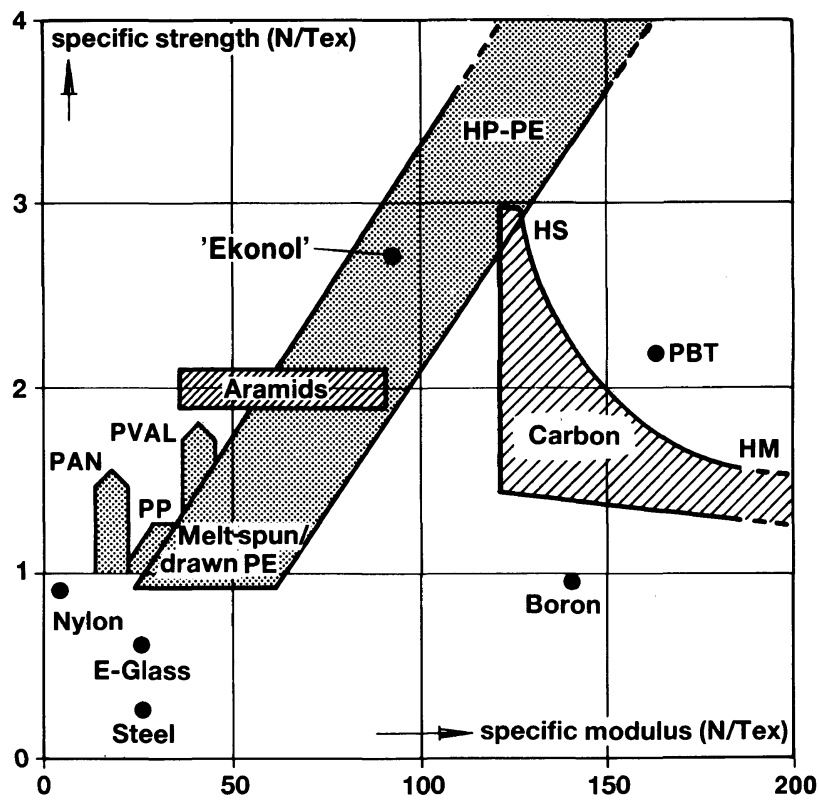

Figure 1. Specific strength vs. specific modulus of fibres.

ly to obtain chain-extension (for a review of various techniques and attempts, see ref 4). The gel-spinning process, invented in the late seventies at DSM-Research ${ }^{5}$ and currently in development by Dyneema-Vof ${ }^{6}$ (a joint venture of DSM and Toyobo) proved to be most versatile and successful in producing chain-extended polyethylene structures including fibres, tapes and films. Since the invention of the gel-spinning process, several (patent) publications describing variations of this process have appeared, ${ }^{7-9}$ but the basic principles of the invention have not been challenged. The gel-spinning process comprises the spinning/extrusion of semidilute solutions of high-molecular-weight polyethylene, $\bar{M}_{w}>4 \times 10^{5}$, followed by ultradrawing of the as-spun quenched filaments, either wet or dry. In-line spinning/drawing can be performed for continuous production of PE fibres with exceptional properties. Figure 1 shows the specific strength/ modulus of HP-PE fibres (HP = high-performance) and shows the potential of producing a large variety of strength/stiffness combina- tions to match the requirements of the user.

Gel-spinning of other flexible polymers have been explored as well, such as polypropylene (PP), polyacrylonitrile (PAN) and poly(vinyl alochol) (PVAL), for details, see ref 4 . However, as in Figure 1, HP-PE is undoubtly the "primus inter pares" at this point in time.

Various explanations have been proposed to explain the beneficial effect of solutionspinning/casting upon the subsequent drawing behavior (see for a detailed discussion ref 4). However, the original explanation given by Smith et al. ${ }^{10}$ — "the strongly enhanced drawability is due to a reduction of the entanglement density" - proved to be most versatile. In this model it is assumed that trapped entanglements in the as-spun/cast fibres or films act as physical crosslinks that are semi-permanent on the time scale of the subsequent drawing experiment. In case of melt-crystallized UHMWPE the high entanglement density is prohibitive for ultra-drawing. Dissolution however, decreases the number of trapped entanglements per chain in the as-spun yarns or castfilms and this favorable situation is preserved 
upon spinning/quenching into the as-spun yarns resulting in an increased drawability. The maximum draw ratio $\lambda_{\max }\left(l / l_{0}\right)$ could be related for isothermal drawing experiments to the initial polymer concentration as follows:

$$
\lambda_{\max }=C \Phi^{-0.5}
$$

Equation 1 could be explained semiquantitatively by a reduction of the entanglement density proportional to the degree of dilution, see ref 10. Entanglements as such are rather elusive species in polymer science which does not prevent many polymer scientists to invoke their presence as important to explain various effects observed in rheology and rubber elasticity.

In this paper we discuss some recent experimental results in order to specify or at least to attempt to indicate the nature of constraints controlling ultra-drawability of solution-spun/ cast UHMW-PE.

\section{EXPERIMENTAL}

\section{Materials}

Various UHMW-PE grades were studied. The samples were obtained from regular producers such as Hoechst/Ruhrchemie (Hostalen Gur-412), Himont (Hifax-1900) and Mitsui (Hizex-240 M): $\bar{M}_{w}$ 's typically around $1.5 \times$ $10^{3} \mathrm{~kg}$ mole $^{-1}$. Solution-cast films, used for drawing experiments, were obtained by dissolving batchwise UHMW-PE in decalin. A suspension of UHMW-PE/decalin in a flask, nominal concentration usually $1.5 \% \mathrm{w} / \mathrm{v}$, was transferred into a preheated silicon-oil bath, $T$ approx. $160^{\circ} \mathrm{C}$. The suspension was stirred to obtain a homogeneous dispersion and when dissolution starts at around $160^{\circ} \mathrm{C}$, as shown by agglomeration of powder particles and the "Weissenberg effect" around the stirrer, stirring was stopped and the system was left to heat to approx. $160^{\circ} \mathrm{C}$ ( $T$-setting of oil bath). The solutions were subsequently held at this temperature (ripening) for approx. $2-5 \mathrm{~h}$ to obtain (macroscopically) homogeneous solu- tions.

To obtain films suitable for ultra-drawing experiments, the solutions were poured into stainless-steel and/or aluminium trays. Crystallization/gelation occurs and the solvent decalin evaporates from the system which takes about 2-3 days at room temperatures.

Deuterated PE (D-PE), purchased from Merck, Sharp, and Dohme, $\bar{M}_{w}$ approx. $5 \times 10^{2} \mathrm{~kg} \mathrm{~mol}^{-1}$ was used in the SANS studies. The D-PE was dissolved in decalin and crystallized to obtain a fine dispersion of crystals. After cooling to room temperature, UHMW-PE powder was added; overall concentration of mixture D-PE $+\mathrm{H}-\mathrm{PE}$ is approx. $2 \%$ in decalin. The same film-casting procedure was adopted as described above.

Solution-cast films were extracted after evaporation of decalin to remove the residual traces of solvent. In order to remove voids in the sample (for SAXS, WAXS, and SANS studies), the cast-films were pressed at approx. $90-100^{\circ} \mathrm{C}$ at $2-10 \mathrm{MPa}$.

Compression-moulded UHMW-PE films were obtained by pressing the as-received powder samples at $200^{\circ} \mathrm{C}$ for $15-60 \mathrm{~min}$ (depending on the thickness), and subsequently quenching to room temperature.

DBPC was usually added as stabilizer in all cases, concn. $0.1-0.3 \%$ based on polymer.

In the text, $R$ refers to compressionmoulded and $S$ to solution-cast samples.

\section{Techniques}

Drawn tapes were produced by either stretching cast-films on "hot-shoes" manually, using ink-marks to determine the draw ratio $\lambda$, or by drawing in a tensile tester (Zwick, Instron) equipped with an oven. In the latter case strain-transducers were employed in recording stress-strain curves.

Drawing above the melting temperature was performed in a Göttfert-Rheostrain using silicon oil with matched density to polyethylene as heating medium, strain rate approx. $0.1 \mathrm{~s}^{-1}$.

Suspensions of UHMW-PE in decalin and 
paraffin oil were extruded in a laboratory scale co-rotating twin screw extruder, using a gear pump to control the throughput/residence time, see refs 17 and 18 for further details.

Crosslinking of as-spun/cast fibres and films was performed via EB-radiation using a Van der Graaff generator operating at $3 \mathrm{MV}$ in the G.B.S.-station of Dr. Wiesner/Wiehl-FRG. Prior to radiation solvent was removed via extraction.

WAXS and SAXS studies were usually performed on identical samples using various camera's (Kiessig, Statton). Ni-filtered, $\mathrm{Cu}-K_{\alpha}$ radiation was used and generated at $40 \mathrm{kV} /$ $25 \mathrm{~mA}$. Sample to film distance, $400 \mathrm{~mm}$ (SAXS) and $67 \mathrm{~mm}$ (WAXS), respectively.

The WAXS studies performed related to the hexagonal phase were performed with stretched fibres. The filaments were embedded in epoxy, Europox 730/XE 278, to constrain the structure. The composite structure was wrapped in aluminium for thermal conductivity. The temperature as indicated in this paper is $\pm 2{ }^{\circ} \mathrm{C}$ accurate in these experiments.

Transmission-electron-microscopy was per- formed using a Philips EM-400. For details of sample preparation see ref 11 .

Small-angle-neutron-scattering (SANS) studies were performed at $\operatorname{Ris} \Phi$-Denmark by Dr. M. Stamm of M.P.I./Mainz. Details about SANS experiments and data analysis will be published in a forthcoming paper. ${ }^{12}$

\section{RESULTS AND DISCUSSION}

\section{Ultradrawability}

Figure 2 shows the well-known stress-strain curves for respectively melt-crystallized $(R) v s$. solution-crystallized $(S)$ UHMW-PE. These stress-strain curves are typical and illustrative to show the dramatic effect of crystallization conditions/history on the drawability of polyethylene in the solid state. Figure 2 shows in fact the essence of the invention of the gel-spinning process for UHMW-PE, being that solution-cast/spun ultra-high-molecularweight PE is ultra-drawable in the solid state, even after complete removal of solvent. The role of the solvent is to remove "the constraints" which limit the drawability of melt-crystallized

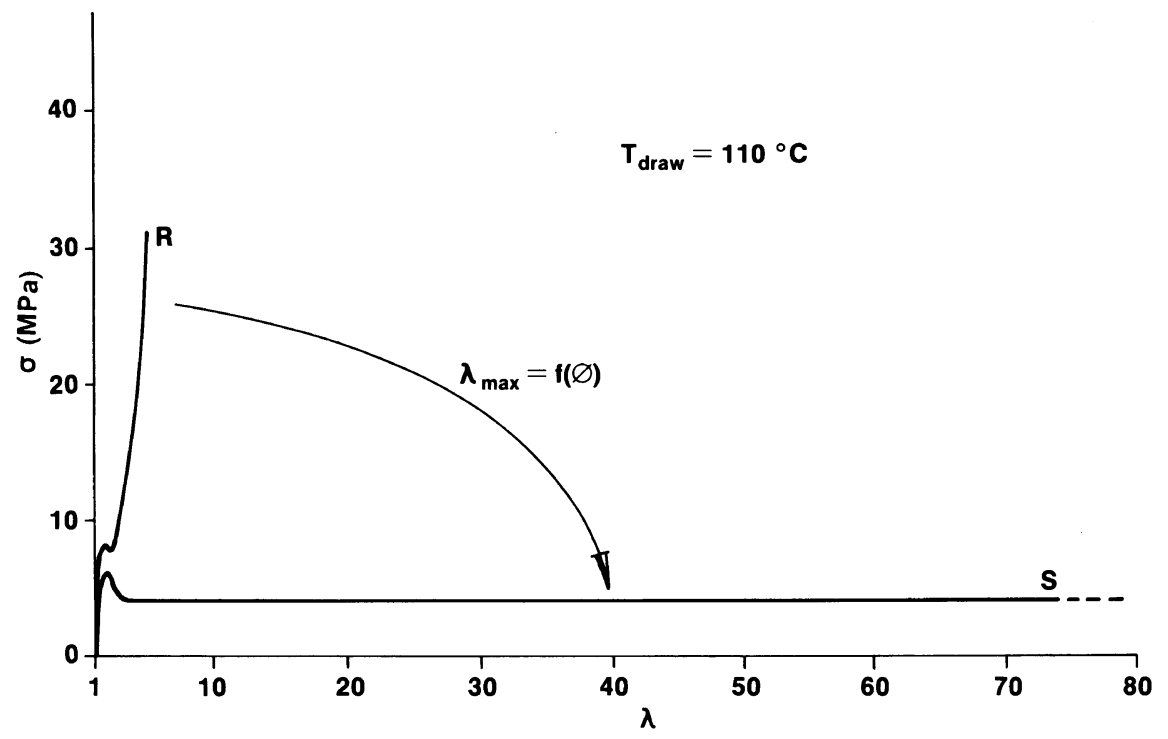

Figure 2. Stress-strain behavior of respectively compression-moulded $(R) \quad v s$. solution-crystallized UHMW-PE $(S)$. In this case $S$ corresponds to a cast film of Hostalen-Gur 412 from a $2 \%$ decalin solution. Residual solvent was extracted prior to drawing. 

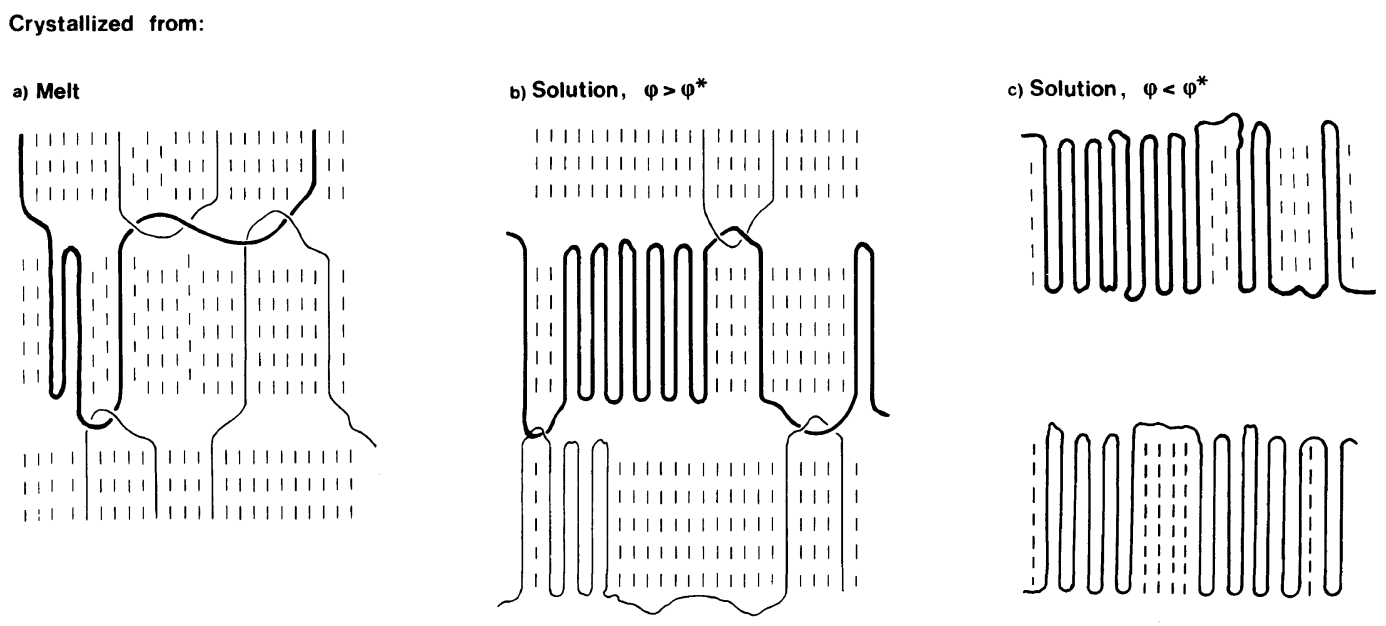

Figure 3. Chain topology as a function of crystallization conditions [reproduced from P. J. Lemstra and R. Kirschbaum, Polymer, 26, 1372 (1985)].

UHMW-PE's and to induce a favourable structure for ultra-drawing. Once this structure is laid down, the solvent can be removed by extraction and/or evaporation prior to the drawing process provided that this favourable structure is not destroyed by (partial) melting/ re-crystallization or re-dissolution.

Gelspinning of UHMW-PE or proposed "alternatives" such as suspension-spinning, ${ }^{13}$ melt-kneading, ${ }^{9}$ and xero-gel formation, ${ }^{7}$ are in fact identical processes, comprising two steps:

a. Dissolution of long-chain molecules (removal of constraints/disentangling).

b. Solidification (preservation of dissolution history)/formation of folded-chain crystals and subsequent ultra-drawing (transformation of folded-chain crystals into chainextended structures).

In a previous paper, ${ }^{14}$ trapped entanglements/"constraints" were represented as topologically interlocked macromolecules of the Langley-type, ${ }^{15}$ see Figure 3.

In order to disentangle the long-chain molecules $\left(\bar{M}_{w}\right.$ of UHMW-PE is above $10^{3} \mathrm{~kg}$ $\mathrm{mol}^{-1}$ ), relatively long dissolution times are expected to obtain homogeneous solutions which are suitable to be used as spinningdopes or for casting films possessing ultradraw characteristics, even at low concentrations (see for instance the ASTM-index for measuring the intrinsic viscosity of UHMW$\left.\mathrm{PE}^{16}\right)$.

Surprising however, ultra-drawable UHMW-PE structures can be generated on a short time scale, for example via extrusion or swelling. ${ }^{17,18}$

Figure 4 shows an experimental set-up for the continuous production of HP-PE fibres, see also ref 17 . The ultra-drawability of the asspun yarns can easily be determined by sampling the yarns before the drawing operation, extracting residual solvent, and performing stress-strain measurements similar to drawing cast-films as shown in Figure 2. A residence time of several minutes in the extruder (see ref 17 for description of extruder) is sufficient to obtain ultra-drawable extrudates. ${ }^{*}$

Another way to generate ultra-drawable UHMW-PE structures, not related to gel-

* In the actual practice of continuous spinning and multi-stage drawing much higher $E$-values are obtained. However, technological aspects are outside the scope of the present paper. 


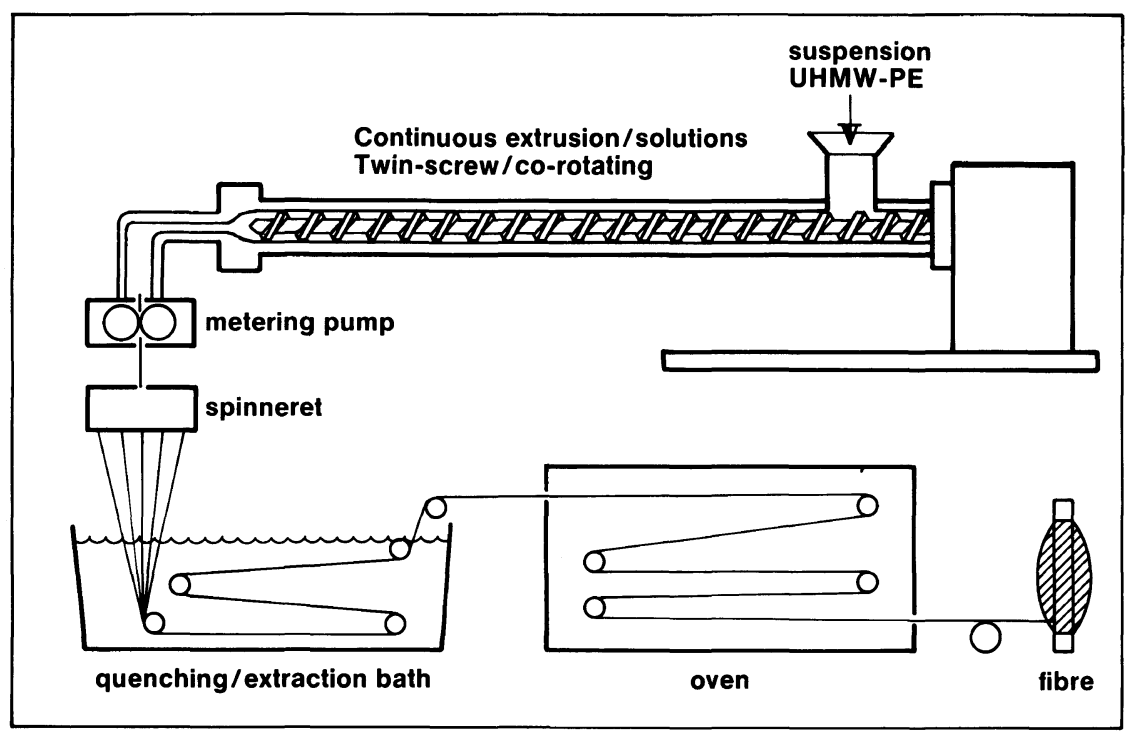

Figure 4. Experimental set-up for the continuous production of HP-PE fibres.

spinning but to demonstrate the short time span, is swelling of compression-moulded UHMW-PE. ${ }^{18,22}$ Table I shows that soaking a compression-moulded film or sheet for a short time in hot decalin renders an ultra-drawable precursor. The limiting factor seems to be the diffusion of solvent rather than an anticipated time consuming process of unravelling entangled long chains.

In both experiments, extrusion and swelling, it is essential that the system is heated to above the dissolution temperature and the maximum draw ratio increases with respectively a decreasing polymer concentration in solution (extrusion) and an increasing solvent uptake (swelling). Therefore these experiments do not violate the concept of trapped entanglements but demonstrate that ultra-drawability can be generated quickly once the macromolecule, or its segments, have experienced the presence of a sufficient amount of solvent.

The short time-scale involved in transforming UHMW-PE from a draw-resistant into an ultra-drawable material, has its counterpart in melting/recrystallization. The ultra-drawability of solution-cast UHMW-PE can be
Table I. Swelling/drawing of compressionmoulded UHMW-PE

\begin{tabular}{|c|c|c|c|}
\hline $\begin{array}{c}\text { Sample } \\
\text { thickness }\end{array}$ & $\begin{array}{l}\text { Residence-time } \\
\text { in decalin } \\
\text { at } 160^{\circ} \mathrm{C}\end{array}$ & $\begin{array}{c}\lambda_{\max } \\
\left(110^{\circ} \mathrm{C}\right)\end{array}$ & E-Modulus \\
\hline $\mathrm{mm}$ & s & & \\
\hline 0.5 & 900 & 50 & 90 \\
\hline 0.1 & 40 & 70 & $>100$ \\
\hline
\end{tabular}

lost almost instantaneously by heating the sample above the melting temperature and recrystallization by quenching. In a previous paper $^{14}$ it was shown that heating above $T_{\mathrm{m}}$ for approx. 60 seconds was sufficient to destroy the ultra-drawability and the molten/ re-crystallized sample in indistinguishable from a straight forward compression moulded sample. The same effect is even observed for a crosslinked sample. EB-radiation of solutioncast films can produces permanent chemical crosslinks but depending on the radiation dose the ultra-drawability can be preserved to a large extent, see Figure 5 and ref 19. If these cross-linked films are heated above $T_{\mathrm{m}}$ and 


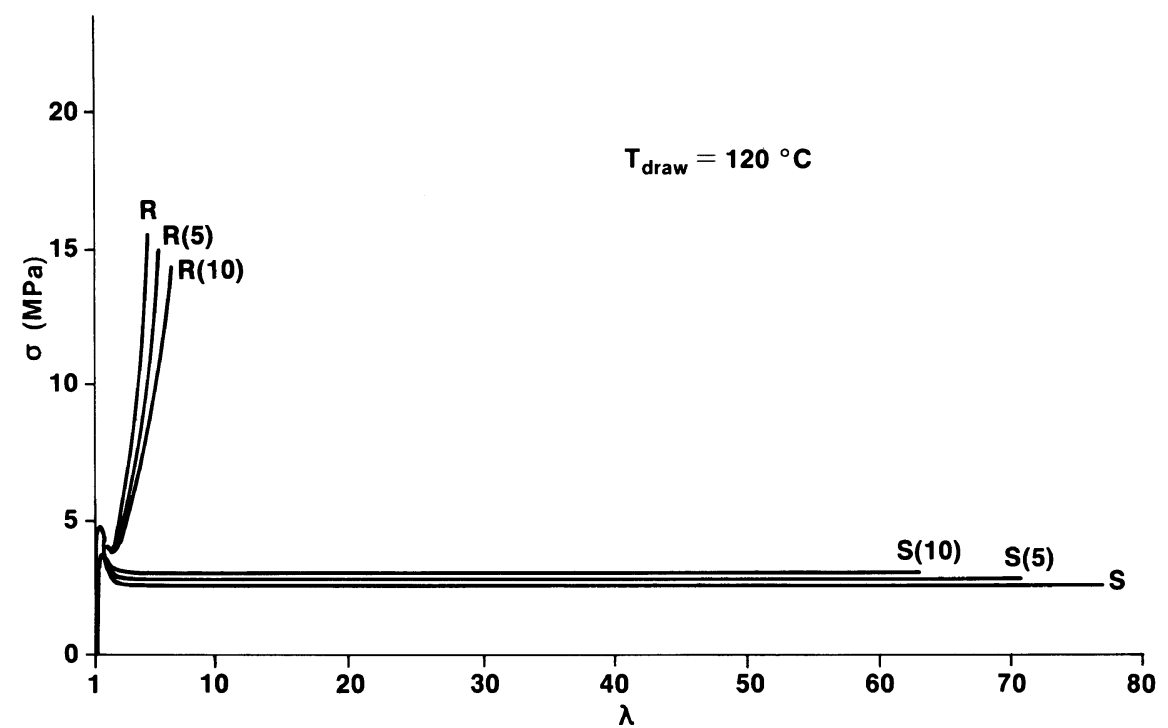

Figure 5. Stress-strain behaviour of EB-radiated/crosslinked solution-cast UHMW-PE films. $S=$ solution-cast UHMW-PE (Hostalen Gur), initial concn. $1.5 \% ; S(5)$ and $S(10)$ are radiated cast-films, respectively 5 and 10 MRAD. $R(5)$ and $R(10)$ refer to radiated and subsequently re-crystallized films (heated to $150^{\circ} \mathrm{C}$ for about 2 minutes) whereas $R$ is the usual compression-moulded sheet.

recrystallized, the ultra-draw characteristics vanish almost instantaneously.

The results discussed above are less compatible with a model of trapped entanglements controlling drawability. The ease of generating ultra-drawability and the corresponding rate of losing this favourable property, suggest that local re-arrangement of chain segments can modulate the drawability of UHMW-PE in the solid state.

\section{Some Comments on the Drawing Mechanism}

Figure 6 shows a series of WAXS, SAXS, and SANS patterns as a function of $\lambda$. For this particular experiment cast films of UHMWPE were used (see EXPERIMENTAL section). The cast films consist of stacked lamellar crystals as shown Figure 7. In case of WAXS/ SAXS studies, the incident X-ray beam was respectively parallel and perpendicular to the film surface. The WAXS and inserted SAXS patterns are self-explanatory, at least for the starting material, $\lambda=1$. They reveal the stacking of lamellar crystals (SAXS, parallel) with a long spacing of approx. $120 \AA$ in line with the TEM results of Figure 7.

During isothermal drawing necking occurs and the long spacing initially increases to $200-300 \AA$, depending on the drawing temperature (annealing). After the necking is complete, at $\lambda \sim 6$ in this case, the lamellar crystals have been incorporated as folded-chain clusters with a long spacing in this case of about $300 \AA$, in microfibrillar units.

During further drawing chain-extension occurs and folded-chain clusters unfold gradually into extended-chain structures as can be inferred qualitatively from the anisotropy in the SANS patterns at higher draw ratios. The long spacing during the transformation from folded-chains to extended-chains remains more or less constant as can be observed also in the SNAS patterns. At high draw ratios, the long spacing disappears.

The authors hasten to remark that the drawing process of semi-crystalline polymers has been studied extensively before, notably by Peterlin. ${ }^{20}$ Figure 6 does not show new results 
WAXS/SAXS and SANS patterns of cast UHMW-PE films as $f(\lambda), T_{\text {draw }}=115^{\circ} \mathrm{C}$
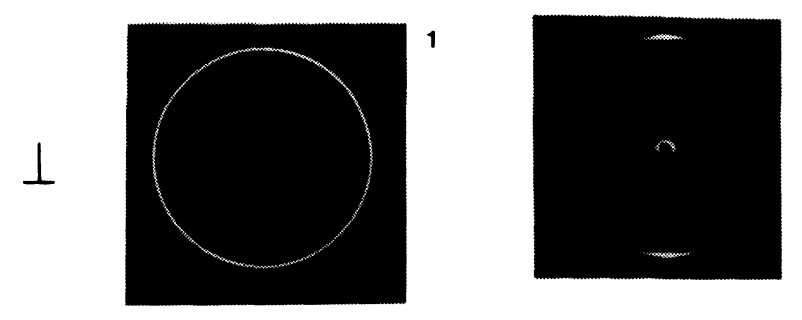

6
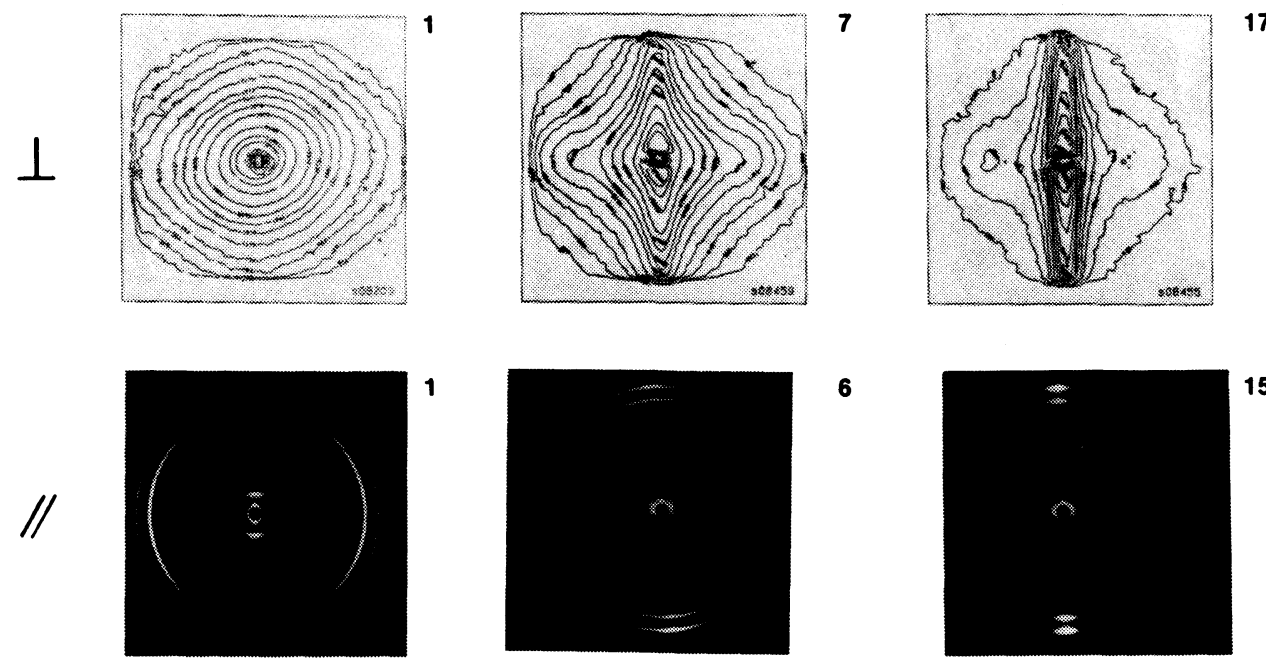

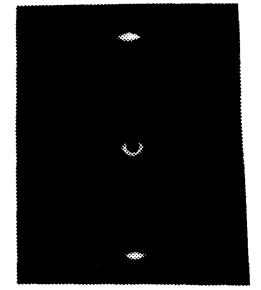

15

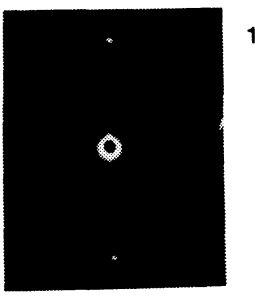

100

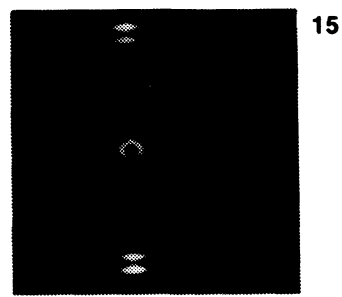

Incident beam $\perp$ and // to film surface, drawing direction horizontal, draw ratios are indicated (upper right corner)

Figure 6. WAXS, SAXS, and SANS patterns of UHMW-PE as a function a draw ratio. The wavelength of neutrons was $12 \AA$. The draw ratios $(1-100)$ are indicated in the figure. 


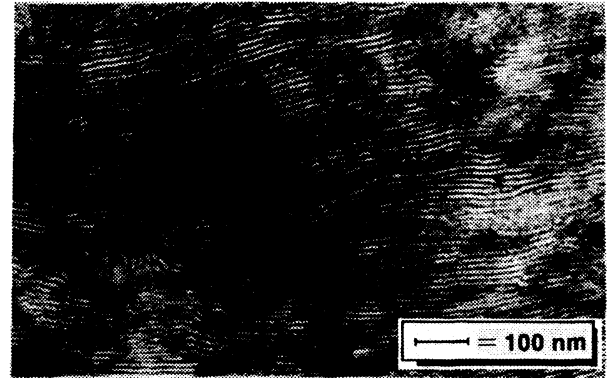

Figure 7. TEM-micrograph of solution-cast UHMWPE film, for experimental conditions, see ref 11.

but merely indicates that UHMW-PE, cast from solution, has a similar drawing behaviour compared to standard PE grades, the difference lying in its extreme large draw ratios and a more perfect chain-alignment. The latter property however is difficult to measure in view of limited resolution and experimental accuracy of SANS experiments, (to be published, ref 12).

According to Peterlin, the number of interlamellar tie-molecules is of prime importance in the drawing process. The interlamellar tie-molecules become intermicrofibrillar tiemolecules during the lamel-fibril transformation and during further draw they are extended. In his view, the number of these interlamellar/microfibrillar tie-molecules determine the drawing stress and the maximum achievable draw ratio. ${ }^{20}$

In the original entanglement-concept as discussed before, trapped-entanglements are located between folded-chain crystals (see Figure 3) and act as semi-permanent physical crosslinks on the time scale of the drawing experiment. Trapped entanglements as such are also of an interlamellar nature.

In view of the results discussed above, in particular the rapid generation and corresponding loss of ultra-drawability, even in the case of crosslinked samples upon recrystallization, another type of constraint should be taken into account in the ultradrawing process of UHMW-PE. In the

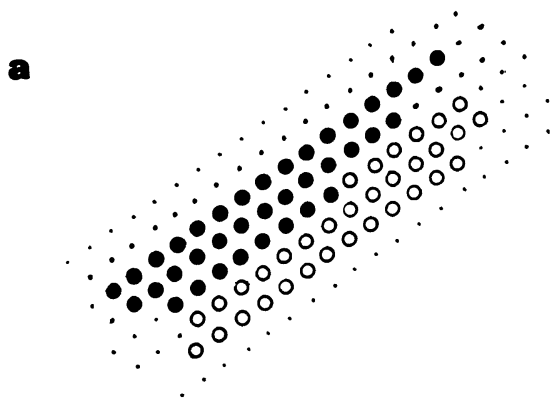

b

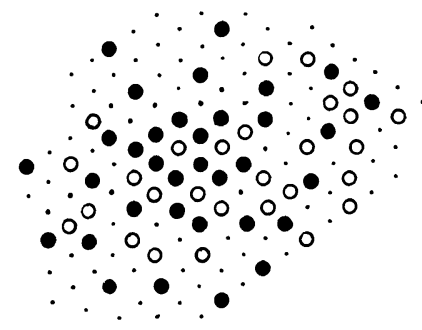

Figure 8. Arrangements of stems in lamellar crystals. The dots represent stems of molecule folding along 110 plane, viewed along $c$-direction. For sake of simplicity no folds are drawn and only a limited number of stems is indicated for two molecules (heavy and open dots): a) adjacent re-entry; b) stem mixing.

entanglement-concept, it was tacitly assumed that UHMW-PE powder is highly entangled and since chains can not cross mutually, entanglements have to be removed to obtain chain-extended structures. At this point in time however, it is questionable whether aspolymerized UHMW-PE (reactor) powder is highly entangled, see page 94 footnote. ${ }^{* *}$ The goal of ultra-drawing UHMW-PE is to transform each individual chain molecule from a folded-chain conformation into a chainextended conformation. In the case of UHMW-PE (mol. weight above $10^{6}$ ), hundreds of stems per molecule have to be accommodated in the (lamellar) crystal lattice. It is clear that adjacent re-entry and 


\section{P. J. Lemstra, N. A. J. M. van Aerle, and C. W. Bastiaansen}

locality of the macromolecules within the lamellar crystal(s) facilitate the process of ultra-drawing which comprises fragmentation of lamellar crystals via shearing, tilting and subsequent unfolding of clusters, see Figure 6. Figure 8a shows schematically the idealized but oversimplified situation of adjacent re-entry within a lamellar crystal.

The fast decay of ultra-drawability upon recrystallization of solution cast UHMW-PE can be understood by a local intermolecular re-arrangement of stems whereafter a situation

Isothermal drawing of UHMW-PE
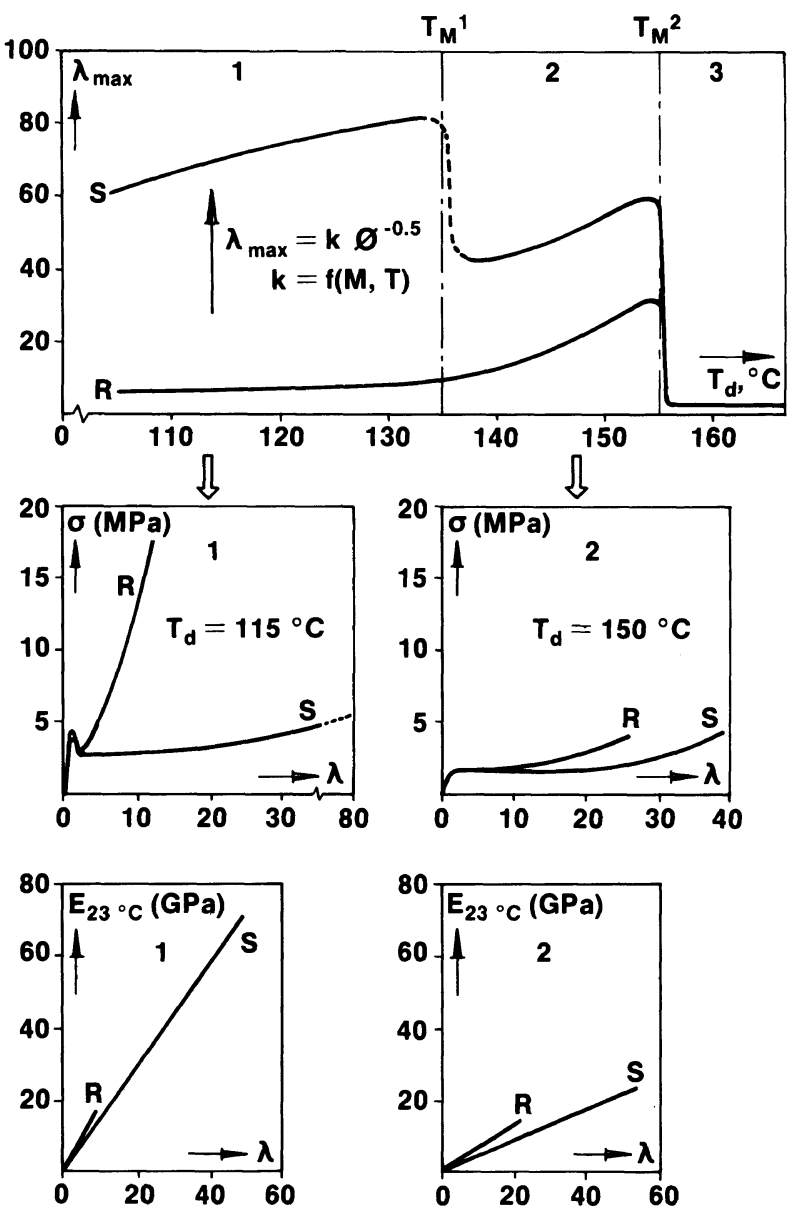

Figure 9. Drawing behavior of UHMW-PE as $f(T)$ showing $\lambda_{\max }$ as $f(T)$ (upper curves). The stressstrain behavior in regions 1 and 2 (middle) and the corresponding properties (bottom).

** During their presentation at the 2nd SPSJ conference, T. Ohama, T. Kanamoto, K. Tanaka, M. Takeda (Science University of Tokyo), and R. S. Porter (Univ. of Massachusetts, Amherst), showed that some commercial UHMW-PE powders could be processed in the solid state via solid-state coextrusion into extrudates which are highly drawable. This implies that as-polymerized UHMW-PE powders are not necessarily highly entangled as a consequence of the polymerization procedure: heterogeneous catalyst system and growing chains which precipitate during polymerization in the form of lamellar or partly chain-extended structures depending on the polymerization conditions. The influence of polymerization conditions on the (ultra)draw behaviour of UHME-PE has been discussed before by Smith et al., ${ }^{21}$ the concept of "virgin polyethylene." 
is obtained of intermixed stems, see Figure 8b. Upon heating solution-cast UHMW-PE above the melting temperature, a relative small displacement of stems can create a situation which is completely different from the original intralamellar arrangement of stems and less suitable for ultra-drawing.

Summarizing, during deformation of UHMW-PE in the solid state the intralamellar arrangement of stems could play an important role in the drawing process. Crystallization from solution promotes adjacent re-entry and separation of macromolecules within the lamellar crystals. Crystallization from the melt or re-crystallization of solution-cast UHMWPE results in mixing of stems (stem dilution) of different macromolecules. In the deformation process of UHMW-PE, stems of different macromolecules impose mutual constraints during the process of fragmentation/shearing of lamellar crystals and subsequent unfolding of the clusters.
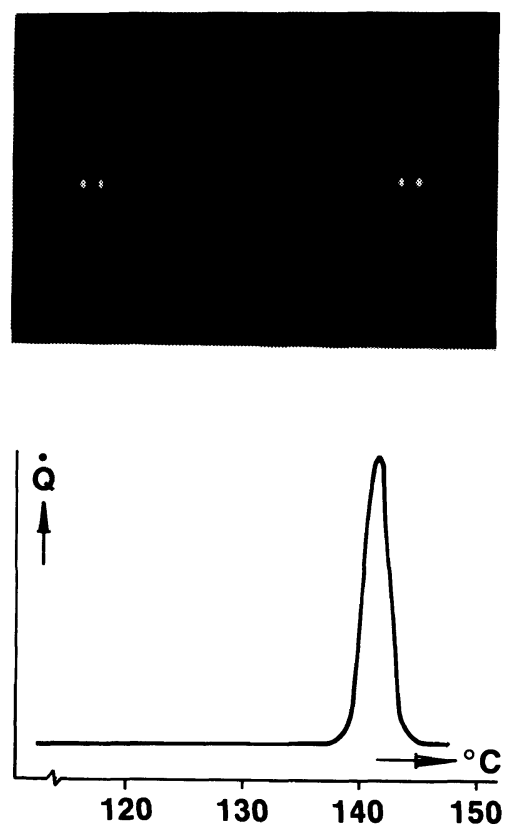

Figure 10. WAXS and thermal behavior of drawn UHMW-PE tapes (draw ratio approx. 30) in respectively region-1 (left) and region-2 (right).
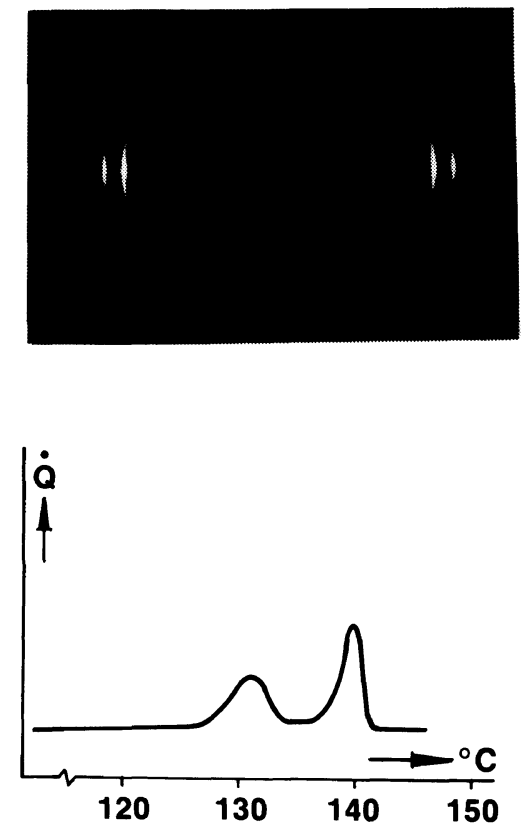

\section{Temperature Dependence of Drawability}

Despite the various models that are proposed and/or techniques which are claimed to be different, the original finding made at DSM that solution-spun/cast UHMW-PE is ultradrawable depending on $\Phi$, is not challenged.

Based on a large series of experiments, we still find $\lambda_{\max }=k \Phi^{-0.5}$, where $k$ is mainly dependent on temperature and molecular weight, at least for isothermal drawing and drawing in the solid state below $T_{\mathrm{m}}^{1}$.

The emphasis on isothermal drawing and the definition of $T_{\mathrm{m}}^{1}$ needs further explanation to illustrate the peculiar drawing behaviour of UHMW-PE and to distinguish from continuous spinning/multi-stage drawing operations.

In case of isothermal drawing, three temperature regions can be discerned as shown in Figure 9.

In region-1, $T<T_{\mathrm{m}}^{1}$, is the temperature range where the drawability is strongly dependent on 


\section{WAXS as $f\left(T,{ }^{\circ} C\right)$}

\section{3}

$\omega$

*

150

4

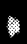

160
152

*

155

4

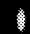

(4)

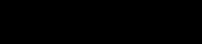

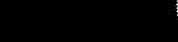

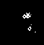

170

Figure 11. WAXS-pattern of drawn UHMW-PE fibre as $f(T)$. The outer ring and streaks originate from Al-coating on fibres. 
the crystallization conditions, $\lambda_{\max } \simeq \Phi^{-0.5} \cdot T_{\mathrm{m}}^{1}$ is the melting point of lamellar crystals, approx. $135^{\circ} \mathrm{C}$. Heating above $T_{\mathrm{m}}^{1}$, followed by re-crystallization into region-1, destroys the ultra-drawability of solution-cast samples and $R$ and $S$ become indistinguishable.

In region-2, $T_{\mathrm{m}}^{1}<T_{\mathrm{draw}}<T_{\mathrm{m}}^{2}$, isothermal drawing is performed in the melt and the large differences with respect to crystallization history are gone. However, small but significant differences can still be observed between meltand solution-crystallized samples, see Figure 9. A tentative conclusion could be that drawing in region- 2 , in the melt, reflects the entanglement density with respect to the stress-strain behaviour in contrast to region-1, solid-state, where local stem arrangements in the lamellar crystals modulate the drawing process.

Drawing in region-2 is less effective, compared to region-1, see Figure 9. During drawing, strain-induced crystallization occurs in region-2 involving the high-molecular weight part of the material (standard linear PEgrades can not be drawn above $T_{\mathrm{m}}^{1}$ ) whereas the lower molecular weight part of the sample relaxes during drawing. The E-modulus, molecular orientation and thermal properties of fibres/tapes drawn in region-2 are poor compared to those drawn in region-1, see Figures 9 and 10.

The upper limit for drawing in region-2 is $T_{\mathrm{m}}^{2}$, approx. $155^{\circ} \mathrm{C}$. This temperature reflects the transition of the orthorhombic into the hexagonal phase. This temperature can be somewhat lower or higher depending on the stress/constraints applied. Figure 11 shows the orthorhombic $\rightarrow$ hexagonal phase as measured on drawn fibres embedded in epoxy. In the hexagonal phase, the system can not sustain an applied stress and fails. Consequently, tensile drawing in region-3 is impossible since if strain-induced crystallization occurs, the (oriented) crystals do not act as stressconnectors.

\section{CONCLUDING REMARKS}

In the previous section, the temperature dependence was discussed for isothermal drawing. In the actual practice of continuousspinning/multi-stage drawing, the difference between region-1 and -2 is not observed. During multi-stage drawing, lamellar crystals are transformed gradually into chain-extended structures along the spinning-line. If the local temperature in the oven does not exceed the local melting temperature of the fibre $T_{\mathrm{m}}^{\mathrm{f}}$, where $T_{\mathrm{m}}^{1}<T_{\mathrm{m}}^{\mathrm{f}}<T_{\mathrm{m}}^{2}$, effective drawing is possible. The maximum drawing temperature is below $T_{\mathrm{m}}^{2}$, the maximum melting temperature of orthorhombic chain-extended PE under tension. By increasing the polymer concentration in solution and optimizing the MWD and the $T_{\text {draw }}$-programme, a unique class of materials can be produced. This is not only reflected in the tensile/stiffness values shown in Figure 1, but also in the transverse and long term (creep) properties, which are superior to those of other chain-extended UHMW-PE structures as for example generated via the surface-growth technique or drawing single crystals of UHMWPE. In the surface growth technique or singlecrystal drawing, dilute solutions of UHMW$\mathrm{PE}$ are used (typically $<1 \%$ ) whereas in the gelspinning process $\Phi$ can be higher by an order of magnitude. The creep behaviour (plateau creep rate) can be modelled in terms of the simple equation for activated flow ${ }^{22}$ :

$$
\dot{\varepsilon}=\dot{\varepsilon}_{0} \exp (-\Delta E / R T) \exp (\sigma \cdot \Delta V / R T)
$$

In eq $2, \Delta E$ is the activation energy, $\Delta V$ the activation volume, $\sigma$ the applied stress and $T$ the temperature. For chain-extended UHMWPE structures, $\Delta E$ and $\Delta V$ are in the range of respectively $25 \mathrm{kcal} \mathrm{mol}^{-1}$ and $20 \AA^{3}$, rather independent of $\Phi$. The frontfactor $\dot{\varepsilon}_{0}$ however is strongly dependent on $\Phi$ for samples of identical MWD and $E$-modulus. With increasing $\Phi$ the material becomes more creepresistant which reflects a favorable and unique 
chain topology in gel-spun/drawn HP-PE fibers.

\section{REFERENCES}

1. W. Carothers and J. W. Hill, J. Am. Chem. Soc., 54, 1586 (1932).

2. W. Fang Hwang, Proceedings of Symposium on Formation, Structures and Properties of $\mathrm{H}-\mathrm{M} / \mathrm{H}-\mathrm{T}$ Fibres. Kyoto, August 1985, p 23.

3. W. Fang Hwang, Proceedings, ISF-85, Hakone, August 1985, p 39.

4. P. J. Lemstra, R. Kirschbaum, T. Ohta, and H. Yasuda, "Developments in Oriented Polymers-2," I. M. Ward, Ed., Applied Science Publishers, London, to be published end of 1986 .

5. DSM/Stamicarbon, NL Patent 79,00990 (1979), NL Patent 79,04990 (1979) and corresponding U. S. Patent 4,344,908 (1982), U. S. Patent 4,422,993 (1984).

6. Japan Chem. Week 12/6-1986.

7. D. C. Prevorsek, "Polymer Liquid Crystals," Academic Press, New York, N. Y., 1983, p 357.

8. Allied Fibres, EP Patent 64167 (1982).

9. Mitsui, EP Patent 115,192 (1984).

10. P. Smith, P. J. Lemstra, and H. C. Booy, J. Polym. Sci., Polym. Phys. Ed., 19, 877 (1981).
11. C. W. M. Bastiaansen, P. Froehling, A. J. Pijpers, and P. J. Lemstra, "Integration of Polymer Science and Technology," A. L. Kleintjens and P. J. Lemstra, Ed., Elseviers Applied Science Publishers, London, 1985, p 508.

12. M. Stamm, N. A. J. M. van Aerle, and P. J. Lemstra, to be published.

13. J. Smook and A. J. Pennings, Polym. Bull., 10, 291 (1983).

14. P. J. Lemstra and R. Kirschbaum, Polymer, 26, 1372 (1985).

15. W. W. Graesley, Advances in Polymer Science, Springer-Verlag, Berlin, 16, Chapter 7, 1974, p 111.

16. ASTM, 35, D1601.

17. NL Patent 85,00428 (Stamicarbon/DSM), 1986.

18. NL Patent 85,00477 (Stamicarbon/DSM), 1986.

19. R. A. M. Hikmet, P. J. Lemstra, and A. Keller, Colloid Polym. Sci., in press.

20. A. Peterlin, "Ultra-High Modulus Polymers," A. Ciferri and I. M. Ward, Ed., Applied Science Publishers, London, Chapter 10, 1979, p 279.

21. P. Smith, H. D. Chanzy, and B. P. Rotzinger, Polym. Commun., 26, 257 (1985).

22. I. M. Ward, "Mechanical Properties of Solid Polymers," John Wiley and Sons, New York, N. Y., 1979, Chapter 8.

23. C. W. M. Bastiaansen, H. E. H. Meijer, N. A. J. M. van Aerle, and P. J. Lemstra, Polym. J., 19, 000 (1987). 